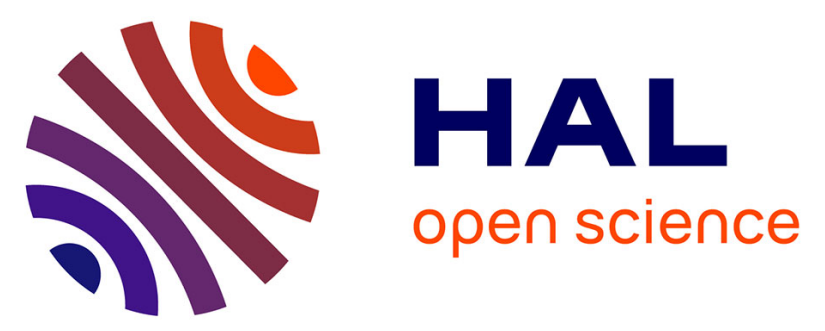

\title{
High-resolution characterization of the diffusion of light chemical elements in metallic components by scanning microwave microscopy
}

\author{
V. Optasanu, E. Bourillot, P. Vitry, C. Plassard, L. Beaurenaut, P. Jacquinot, \\ F. Herbst, P. Berger, E. Lesniewska, T. Montesin
}

\section{To cite this version:}

V. Optasanu, E. Bourillot, P. Vitry, C. Plassard, L. Beaurenaut, et al.. High-resolution characterization of the diffusion of light chemical elements in metallic components by scanning microwave microscopy. Nanoscale, 2014, 6, pp.14932-14938. 10.1039/c4nr04017a . hal-01157263

\section{HAL Id: hal-01157263 \\ https://hal.science/hal-01157263}

Submitted on 17 Nov 2015

HAL is a multi-disciplinary open access archive for the deposit and dissemination of scientific research documents, whether they are published or not. The documents may come from teaching and research institutions in France or abroad, or from public or private research centers.
L'archive ouverte pluridisciplinaire HAL, est destinée au dépôt et à la diffusion de documents scientifiques de niveau recherche, publiés ou non, émanant des établissements d'enseignement et de recherche français ou étrangers, des laboratoires publics ou privés. 


\title{
Nanoscale
}

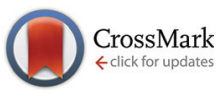

Cite this: Nanoscale, 2014, 6, 14932

\section{High-resolution characterization of the diffusion of light chemical elements in metallic components by scanning microwave microscopy}

\author{
Virgil Optasanu, ${ }^{a}$ Eric Bourillot, ${ }^{* a}$ Pauline Vitry, ${ }^{a}$ Cédric Plassard ${ }^{a}$ Laure Beaurenaut, ${ }^{a}$ \\ Pierre Jacquinot, ${ }^{\mathrm{a}}$ Frédéric Herbst, ${ }^{\mathrm{a}}$ Pascal Berger, ${ }^{\mathrm{b}}$ Eric Lesniewska ${ }^{\mathrm{a}}$ and \\ Tony Montessin ${ }^{a}$
}

An original sub-surface, high spatial resolution tomographic technique based on scanning microwave microscopy (SMM) is used to visualize in-depth materials with different chemical compositions. A significant phase difference in SMM between aluminum and chromium buried patterns has been observed. Moreover this technique was used to characterize a solid solution of a light chemical element (oxygen) in a metal lattice (zirconium). The large solubility of the oxygen in zirconium leads to modifications of the properties of the solid solution that can be measured by the phase shift signal in the SMM technique. The signal obtained in cross-section of an oxidized Zr sample shows the excellent agreement between phase shift profiles measured at different depths. Such a profile can reveal the length of diffusion of the oxygen in zirconium under the surface. The comparison with the oxygen concentration measured by nuclear reaction analysis shows excellent agreement in terms of length of diffusion and spatial distribution of the oxygen. A rapid calibration shows a linear dependence between the phase shift and the oxygen concentration. The SMM method opens up new possibilities for indirect measurements of the oxygen concentration dissolved in the metal lattice.

Received 16th July 2014 Accepted 8th October 2014

DOI: $10.1039 / c 4 n r 04017 a$

www.rsc.org/nanoscale
Measuring their spatial distribution is useful for understanding the mechanisms governing the diffusion or for model verification and parameter determination. The accurate measurement of light elements is not easy and often requires large experimental facilities like the nuclear reaction analysis technique (NRA or nuclear microanalysis). ${ }^{1}$ Moreover, most common techniques proceed to surface measurements (energy dispersive spectrometry - EDS, wavelength dispersive spectroscopy - WDS, X-ray photoelectron spectroscopy - XPS, and Auger electron spectroscopy). These techniques are essentially used for surface analysis because the interactions of the electron beam with the material are limited to a few nanometers under the surface..$^{2-4}$ So, the precise measurement of the concentration of light elements is impossible with most common surfaces techniques especially for very volatile species or when the pollution of the surface occurring during surface preparation cannot be avoided. The best examples are the measurement of hydrogen in metals and oxygen in reactive materials.

Generally, the measurement corresponds to an average of the information obtained on a small volume ( $1 \mu \mathrm{m}^{3}$ for EDS) and includes the contaminated surface, a fact that can present a substantial downside.

In this article we will proceed to accurate measurements of the oxygen concentration by NRA. This method is able to 
obtain information under the contaminated sub-surface. ${ }^{1}$ We will use these measurements for a comparison with results obtained using the new technique proposed here.

Since the invention of scanning probe microscopy, the characterization of surface properties at the nanoscale has been achieved in different types of samples. ${ }^{5-8}$ Among these microscopes, it is certainly the atomic force microscopy (AFM) technique that has experienced the greatest development, potential through various AFM spectroscopy measurement techniques. $^{9-16}$ All these scanning probe microscopes (SPM) give surface information; however many research studies and applications about the behavior of materials require investigations into the sample volume. Experimental techniques such as acoustic or ultrasonic microscopy and scanning thermal microscopy (SThM) give volume information, but these techniques are not usable for all types of samples. ${ }^{17-20}$

In this article we present an original tomography method based on scanning microwave microscopy (SMM). ${ }^{21,22}$

To illustrate this original approach, we will first study the case of a calibrated sample composed of different buried metallic materials, which present very close physical properties. Then, we will focus on the complex case of the solid solution of oxygen in zirconium.

The oxygen dissolved in zirconium produces detectable modifications of the physical and chemical properties of the metal. $^{23}$

The choice of zirconium is driven by the significant solubility of oxygen in this metal, which is about 29 atomic percent. Exposition to a high temperature $\left(>450{ }^{\circ} \mathrm{C}\right)$ leads to an anionic oxidation process and the rate-determining process is the diffusion of $\mathrm{O}^{2-}$ ions into the metal crystallographic lattice. At high temperatures, the length of diffusion can be up to hundreds of $\mu \mathrm{m} .^{23}$

\section{Experimental section}

\section{SMM experimental devices}

The scanning microwave microscope used for the study is an AFM 5600LS from Agilent Technology. The SMM typically operates at microwave frequencies from $300 \mathrm{MHz}$ to $13 \mathrm{GHz}$, which is substantially higher than the frequencies used in traditional SCM (around $900 \mathrm{MHz}$.).

The most important parameter lies in the choice of the conductive probe. The tips used for experiments are SCM-PIT tips of silicon nitride covered with a conductive layer of a Pt-Ir mixture. The scan rate used is $1 \mathrm{~Hz}$ with a scan range of 80 $\mu \mathrm{m}$. The choice of the cantilever determines the number of frequencies used in the experiments and is related to the coupling between the cantilever and the resonant circuit.

\section{EDS measurements}

The EDS measurements have been realized on a Jeol JSM $7600 \mathrm{~F}$ SEM at $10 \mathrm{keV}$ with an SDD X-MAX $80 \mathrm{~mm}^{2}$ EDS device.

\section{Nuclear reaction analysis (NRA)}

The nuclear reaction analysis was performed at the Institut Rayonnement Matière de Saclay, France, and used a Van De Graaff linear accelerator (maximum 3.7 MeV). The nuclear microanalysis is based on the interactions of light energetic ions $\left({ }^{1} \mathrm{H},{ }^{2} \mathrm{H},{ }^{3} \mathrm{He}\right.$ or ${ }^{4} \mathrm{He}$ in the MeV domain) with the material to be characterized. The concentrations of the constituent elements were determined from the spectroscopy of the emitted particles, elastically scattered (RBS, Rutherford Back Scattering) or induced by nuclear reactions (NRA, nuclear reaction analysis) or the induced photons $\mathrm{X}$ and gamma (particle induced X-ray emission, PIXE, or particle induced gamma ray emission, PIGE). NRA is especially devoted to the analysis of light elements and their isotopes, such as oxygen-16 which may be analyzed from the spectroscopy of protons produced by the interaction of a deuteron beam (reaction $\left.{ }^{16} \mathrm{O}\left(\mathrm{d}, \mathrm{p}_{1}\right){ }^{17} \mathrm{O}\right) .{ }^{1}$ The main advantage of NRA is that the information given is independent of the chemical environment so that the accuracy of the determined concentrations is high. In addition, the intrinsic depth resolution of particle spectroscopies enables to get rid of bias induced by the contribution of oxygen surface contamination (native oxides for instance). For the experiments reported in this paper we used a $1.45 \mathrm{MeV}$ deuteron beam and analyzed the reaction ${ }^{16} \mathrm{O}\left(\mathrm{d}, \mathrm{p}_{1}\right){ }^{17} \mathrm{O}$. Lateral resolution is directly related to beam size, which is $3 \times 3 \mu \mathrm{m}^{2}$ in this case. The size of the scanned area was $80 \times 120 \mu \mathrm{m}^{2}$.

\section{Fabrication of the calibrated sample with buried metal patterns}

The nanofabricated-calibrated sample was elaborate starting from a silicon substrate on which matrices of patterns $(30 \mathrm{~nm}$ depth) were filled by two different materials of $20 \mathrm{~nm}$ thickness (aluminum and chromium), before being entirely covered with a $500 \mathrm{~nm}$ silicon layer (Fig. 1a).

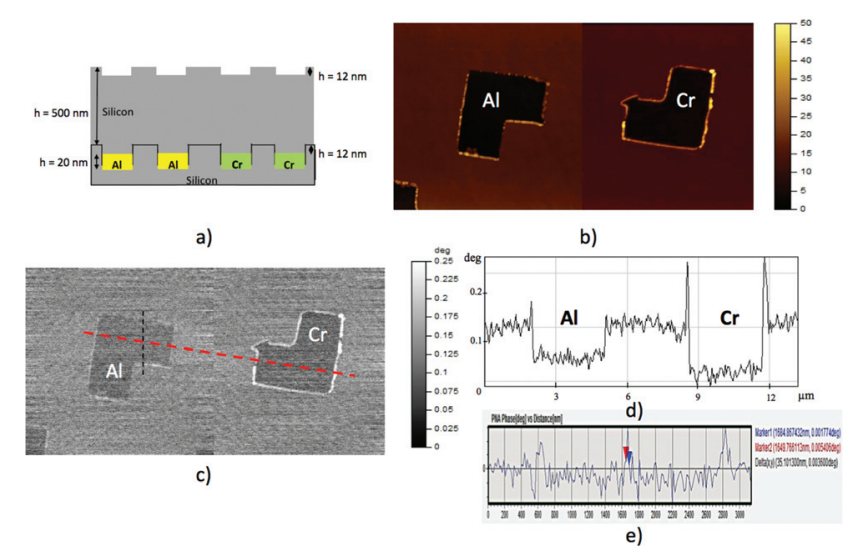

Fig. 1 Calibrated sample with buried metal patterns. (a) Schematic of the buried patterns of Al and Cr. (b) AFM image: topography. Scan size: 16 by $8 \mu \mathrm{m}$. Relative height: $50 \mathrm{~nm}$. (c) SMM phase image ( $f=1.971$ $\mathrm{GHz}$ ). Scan size: 16 by $8 \mu \mathrm{m}$. (d) Cross-section of the phase image revealing the phase difference between the structures of $\mathrm{Al}$ and $\mathrm{Cr}$. (e) Profile of a section carried out on the phase image. Estimation of the lateral resolution (scan size: $8 \mu \mathrm{m}$ ). 
To avoid any confusion between two materials, the Al patterns are laid out in a reversed position compared to those of Cr. Traces of PMMA, partially dissolved by the solvents, remain present after evaporation of the silicon layer is over. It is very difficult to obtain samples without topographical effects on the surface. These residual traces will be used by us later as a reference mark for the investigation of the volume of the sample.

A first characterization (Fig. 1b), at this stage of fabrication, made it possible to measure a difference of $12 \mathrm{~nm}$ between the structures $(\mathrm{Al}$ or $\mathrm{Cr}$ ) and the real height of the patterns.

\section{Preparation of the oxidized zirconium}

For the study of zirconium oxidation, commercial pure zirconium plates (99.2\% Zr from Goodfellow) were used. They were first annealed at $750^{\circ} \mathrm{C}$ under a secondary vacuum for 2 hours and then oxidized in air under atmospheric pressure at $650{ }^{\circ} \mathrm{C}$ for 72 hours. After oxidation the samples were transversely cut and mirror-polished and then analyzed by SEM/EDS, NRA and the SMM technique. Two successive layers cover the pure original metal: a $\mathrm{ZrO}_{2}$ exterior region and an intermediate oxygenenriched metal ( $\mathrm{Zr}-\mathrm{O})$. The oxygen concentration in the enriched zone goes progressively from 29 at.\% to 0 at.\%.

\section{Scanning microwave microscopy theory}

The scanning microwave microscope (SMM) combines the electromagnetic measurement capabilities of a microwave Vector Network Analyzer (VNA N5230A, Agilent Technology) and the nanometer resolution capabilities of an atomic force microscope (AFM). ${ }^{21,22}$ Like all SPM techniques, the SMM gives a nanometer resolution by coupling the local probe effect with the electromagnetic field. Indeed, like near-field scanning optical microscopy (NSOM/SNOM), this resolution is induced by the electromagnetic near field detection to the microwave frequencies. ${ }^{24,25}$ Recently, this non-destructive technique was used to characterize the buried defects in a metal sample. It has been possible to observe the electromagnetic wave absorption properties in the metallic material and to obtain a $3 \mathrm{D}$ tomography of the metallic sample showing these buried inhomogeneities with a nanoscale resolution. ${ }^{21}$

A microwave signal is sent directly from the network analyzer and transmitted through a resonant circuit to a conductive AFM probe that is in contact with the sample being scanned. The cantilever and the probe act as a local radiant antenna for emission and reception of the electromagnetic field. The transmitter-receiver system is limited to interaction between the tip and the surface sample and makes it possible to capture the reflected microwave signal from the contact point. By directly measuring the complex reflection coefficient from the network analyzer, the impedance representing the probe-sample interaction amplitude and the phase at each scanned point can then be recorded, simultaneously with the surface topography.
In the case of the metallic sample, the electromagnetic waves in the range of microwaves have a power of penetration in the sample depending on the frequency used: the electromagnetic wave of high frequency has a lower penetration than a low-frequency wave in a metallic sample. This physical effect corresponds to the skin effect:

$$
\delta=\frac{1}{\sqrt{\pi \mu_{0} \mu_{\mathrm{r}} \sigma f}}
$$

with $\delta$ the skin thickness (in $\mathrm{m}$ ), $\mu_{0}$ the magnetic permeability of the vacuum $\left(4 \pi 10^{-7}\right), \mu_{\mathrm{r}}$ the permeability relating to the conductor, $\sigma$ the electric conductivity (in $\mathrm{S} \mathrm{m}^{-1}$ ) and $f$ the frequency (in $\mathrm{Hz}$ ).

Thus, the potential of the microwave microscope lies in the possibility of both differentiating the microwave images obtained at different frequencies and allowing the establishment of an in-depth cartography. ${ }^{21}$ The microwave images will provide information about the differences in phase, directly related to the nature of the material, obtained at various frequencies.

The expression of the wave reflected through a conductor is written as

$$
E_{\mathrm{r}}=E_{\mathrm{r} 0} \mathrm{e}^{\left(\mathrm{i}\left(\omega t-\frac{z}{\delta}\right)-\frac{z}{\delta}\right)}
$$

with $E_{\mathrm{r}}$ the reflected electric field, $E_{\mathrm{r} 0}$ the attenuated reflected electric field, $\omega$ the pulsation $(\omega=2 \pi f), t$ the moment considered, $z$ the component of the direction of propagation and $\delta$ the skin effect.

The phase $\phi$ of the wave defined by eqn (2) is

$$
\varphi=\left(\frac{z}{\delta}\right)
$$

Thus, in a constant observation point $z$ and for a given frequency, the phase will be modified if the skin effect varies. Based on eqn (1), the skin effect is dependent, at constant frequency, on two factors: the permeability $(\delta$ decreases with increasing permeability) and conductivity ( $\delta$ increases with decreasing conductivity) of metal materials.

\section{Study of a calibrated sample}

The objectives of this first study are to investigate the potentiality of scanning microwave microscopy to probe the depth of samples and also the capability to differentiate two or more types of materials present in the sample.

To control the capability of SMM to differentiate two types of materials with close electromagnetic properties, a nanofabricated-calibrated sample with buried metal patterns (aluminum and chromium) has been developed.

Knowing the electric conductivities and magnetic permeabilities of the two materials ( $\mathrm{Al}$ and $\mathrm{Cr}$ ) and considering that those remain constant in the frequency range used (1-6 GHz), it is possible to express, for a frequency $f$ and the same component $z$, the depth penetration and the phase relative to each material. 
For the Al structures:

$$
\varphi_{1}=\left(\frac{z}{\delta_{1}}\right) \quad \text { where: } \delta_{1}=\frac{1}{\sqrt{\pi \mu_{0} \mu_{1} \sigma_{1} f}}
$$

with the relative permeability $\mu_{1}=1$ and the electric conductivity $\sigma_{1}=3.5 \times 10^{7} \Omega^{-1} \mathrm{~m}^{-1}$.

For the Cr structures:

$$
\varphi_{2}=\left(\frac{z}{\delta_{2}}\right) \quad \text { where: } \delta_{2}=\frac{1}{\sqrt{\pi \mu_{0} \mu_{2} \sigma_{2} f}}
$$

with the relative permeability $\mu_{2}=1$ and the electric conductivity $\sigma_{2}=3.8 \times 10^{7} \Omega^{-1} \mathrm{~m}^{-1}$.

By comparing the penetration depth through the two materials (4) and (5) we obtain:

$$
\frac{\delta_{1}}{\delta_{2}}=\sqrt{\frac{\sigma_{2}}{\sigma_{1}}}
$$

Therefore, we can write:

$$
\frac{\varphi_{1}}{\varphi_{2}}=\frac{\delta_{2}}{\delta_{1}}<1
$$

Then, we realized SMM phase measurements for a frequency of $1.971 \mathrm{GHz}$. According to the theory (eqn (7)) we perfectly check on the SMM phase image (Fig. 1c) that the dephasing introduced by chromium is higher than that for aluminum, presenting a phase image with a greater contrast. From the cross-section (red dashed line, Fig. 1d), we can estimate that this dephasing introduced by chromium is about two times higher than that for aluminium. Thus, it is possible to identify, using the microwave image phase signal given by SMM, the nature of materials present in the patterns. We can notice that the buried materials used present only a 0.3 difference of conductivity, which permits us to underline the good sensitivity of this technique.

Another important parameter of the scanning microwave microscope lies in its resolution, lateral and in depth. The resolution of the microscope could be estimated while measuring, directly on the SMM images, the width of the outline on the buried $\mathrm{Al}$ pattern. On the phase image (Fig. 1c), a profile (black dashed line) was carried out while placing the two cursors with the middle height of the peak representing the outline of the patterns (Fig. 1e). The result shows a resolution close to $35 \mathrm{~nm}$, knowing that it can be still improved for recorded images with smaller buried calibrated patterns. In depth, the result shows that SMM is sensitive to a buried defect whose thickness is about $20 \mathrm{~nm}$.

\section{A complex case: diffusion of a light element in a metal}

Now let us consider the complex case of zirconium oxidation.

The driven process in the oxidation of zirconium at high temperature is the diffusion of the oxygen atoms in the metal lattice up to 29 atomic percent and then transformation into
$\mathrm{ZrO}_{2}$. According to the theory of diffusion, in the $\mathrm{Zr}-\mathrm{O}$ zone the oxygen concentration given by the diffusion processes has a complementary error function (erfc) shape. This variation will then be the same at several depths under the scanned cross-section surface (which is perpendicular to the oxidized face) except on the first few hundreds of nm, where the atmospheric oxygen contaminates the sub-surface during the preparation of the cross-section. Zirconium is well known for its high reactivity with oxygen, which causes inevitable pollution during sample preparation. This implies weak precision in the measurement of the diffusion length with surface analysis techniques and makes it impossible to measure low values of oxygen concentration.

The EDS/SEM and NRA analyses were first performed on the sample used to measure the oxygen quantity in the samples and then compared to the SMM results.

Fig. 2a presents a schematic of the sample and the crosssection used for analysis. Fig. 2b shows the concentration of the oxygen in the sample in atomic percent, obtained by EDS and NRA along a line on the cross-section perpendicular to the oxide-metal interface. For EDS, the analyzed volume is about $1 \mathrm{\mu m}^{3}$ and contains the sample surface, including the contaminated sub-surface. Due to the high affinity of the zirconium to the oxygen, the surface has been inevitably enriched with atmospheric oxygen during the cross-section preparation. This results in a large quantity of oxygen content in the first hundreds of nm under the scanned surface and can mask the original quantity present in the sample before the polishing process. The contamination of the surface with atmospheric oxygen and the overevaluation of the light chemical elements by the EDS technique explains the apparent $12 \%$ oxygen concentration obtained in the pure metal zone. The residual apparent quantity of oxygen on the pure $\mathrm{Zr}$ can affect the measure of oxygen-enriched area and cannot reveal regions with low oxygen content. Given the oxygen pollution of the surface we can expect that the length of diffusion measured by EDS can be under-evaluated. The NRA measurements of the oxygen concentration (see the Experimental section for

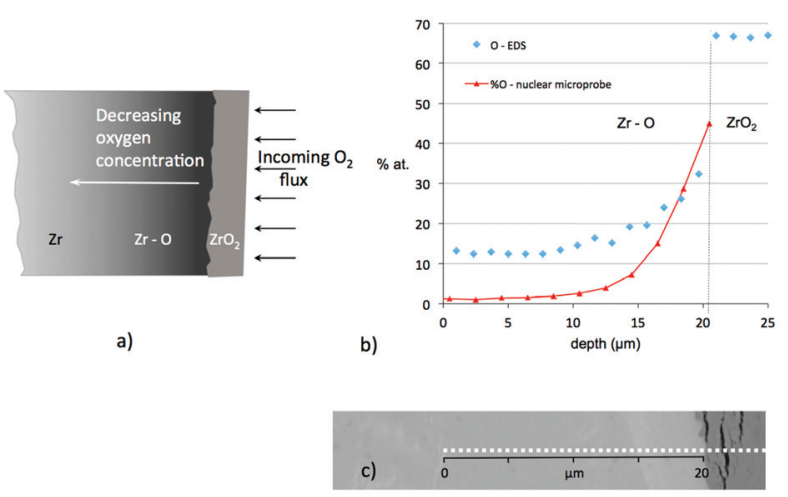

Fig. 2 Analysis and composition of the zirconium sample enriched in oxide. (a) Schematic of the analyzed sample. (b) EDS and NRA measurements of the oxygen level. (c) Electronic microscope image of the analyzed area. 
measurement details) presented in Fig. $2 \mathrm{~b}$ are very accurate because with this technique one can eliminate the response of very superficial layers. The oxygen content in the pure $\mathrm{Zr}$ region is correctly measured and is close to zero. As expected, a longer diffusion region is found, compared to EDS measurements. The spot size in NRA is about $3 \mu \mathrm{m}$ and, close to the interface, it covers both $\mathrm{Zr}-\mathrm{O}$ and $\mathrm{ZrO}_{2}$ zones. It explains the apparent 45 at.\% oxygen content at the interface location. We will use the NRA measurements to compare with the SMM results.

To perform the SMM measurements, we chose a frequency range so as to make a profile investigation inside the sample to lie below the surface layer of pollution. Taking as the reference the deep layer of pure $\mathrm{Zr}$, with the conductivity parameter $\sigma=$ $2.36 \times 10^{6} \Omega^{-1} \mathrm{~m}^{-1}$, the frequencies $11.83 \mathrm{GHz}, 6.87 \mathrm{GHz}$ and $2.21 \mathrm{GHz}$ allow an in-depth investigation between 3 and 7 microns under the sample surface. Then we avoid the influence of the contaminated surface as shown in the scheme of Fig. 3. We focus in this study mainly on the oxygen-enriched metal zone.

The SMM analysis of the same sample gives simultaneously the topographical surface of the sample (in its conventional mode AFM) and the variations of material properties by the measurement of the microwave signal phase shifts (in its SMM mode). The topographical surface image and the SMM images (phase shifts) for several frequencies can be seen in Fig. 4.

Fig. 4a presents the topographic cartography obtained from the amplitude signal and the profile along the dashed horizontal line. The cartography clearly reveals the grains of the metal. The profile shows the roughness of the sample along a line. One can see that the $\mathrm{ZrO}_{2}$ region shows a different roughness from the $\mathrm{Zr}-\mathrm{O}$ and $\mathrm{Zr}$ zones. The amplitude of the roughness is about $20 \mathrm{~nm}$, which is very low compared to the size of the scanned zone, $80 \mu \mathrm{m}$. One can affirm that the surface is flat.

The phase shift for several frequencies can be seen in Fig. $4 \mathrm{~b}-\mathrm{d}$. The phase shift images clearly show a change in the phase signal. This variation for the material used here is introduced solely by a conductivity variation of the material, as the relative permeability is equal to 1 for Zr. Only the oxygen dissolution in the zirconium lattice produces variations of the conductivity. It is well known that the mean free path of electrons is influenced by crystal lattice imperfections such as structural defects, foreign atoms or thermal agitation of the

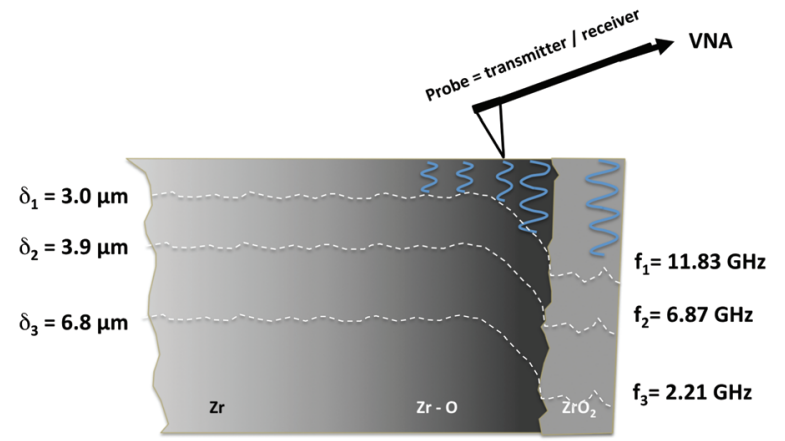

Fig. 3 Principle of SMM measurements on cross-section of an oxidized zirconium sample for different frequencies.
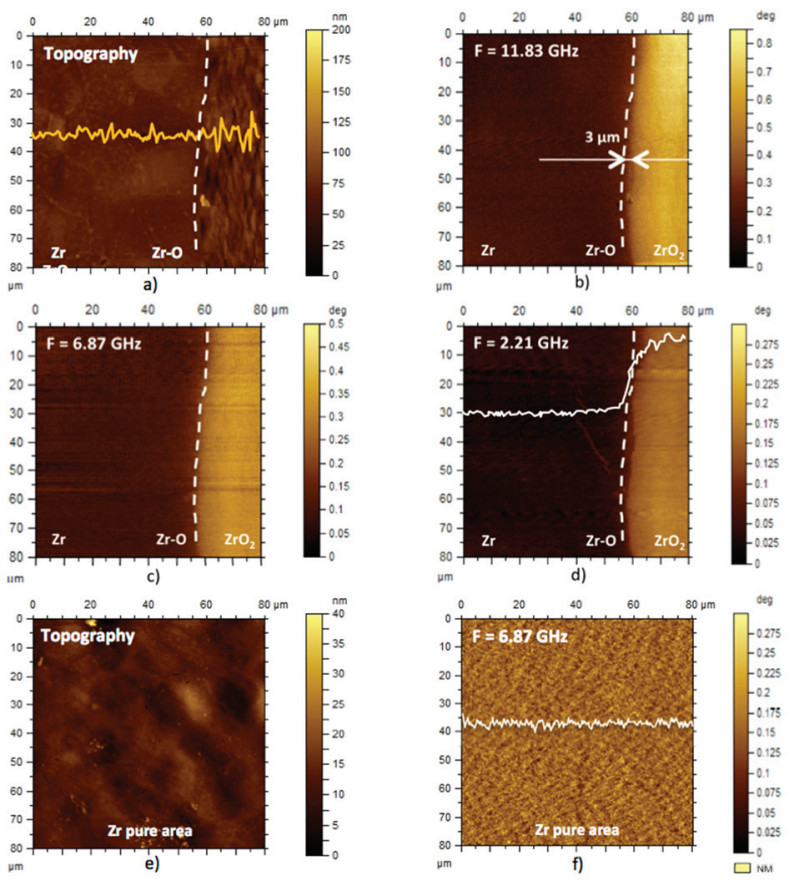

Fig. 4 Topography and phase shift cartography for several frequencies (scan area $80 \mu \mathrm{m}$ ). (a) Topographical image of the sample and a crosssection. (b) Phase shift image at $f=11.83 \mathrm{GHz}$. (c) Phase shift image at $f=6.87 \mathrm{GHz}$. (d) Phase shift image at $f=2.21 \mathrm{GHz}$. (e) Topographical image of pure Zr. (f) Phase shift image of pure Zr at $f=6.87 \mathrm{GHz}$.

ions, and also the electron mobility $\mu$, proportional to the mean free path of electrons, and thus the conductivity is defined by: $\sigma=n q \mu$ with $\sigma$ the conductivity $\left(\mathrm{S} \mathrm{m}^{-1}\right), n$ the number density of electrons and $q$ the elementary charge.

Consequently, the electron mobility decreases as the temperature or the number of faults increases. Then, this phase shift is necessarily linked to a change in composition of the material and highlighted by the SMM technique. This change is the fact that the wave propagates deeper into the oxygenenriched zone (proportionally to this enrichment) in comparison with the area of pure $\mathrm{Zr}$ (Fig. 3).

The position of the oxide-metal interface is materialized by the sudden change in the phase signal within a spatial range less than one micrometer. For a better localization, we used a white dashed line to indicate the position of the oxide-metal interface found on the topographic AFM image (Fig. 4a) on all the phase shift cartographies. The phase shift starts to change close to the interface ( $3 \mu \mathrm{m}$, Fig. $4 \mathrm{~b})$. The interface location can change for different depths as the interface is not perfectly planar. We can observe in Fig. 4b-d these changes. The transition zone between the oxide and the oxygen-enriched metal can be topographically perturbed by the strong difference of hardness of the $\mathrm{ZrO}_{2}$ and $\mathrm{Zr}-\mathrm{O}$ zones, giving rise to differences in the way the polishing/etching technique affects each zone. In this particular case the phase shift signal can also be affected. In our case, a careful polishing allowed us to avoid these effects.

The variations of the phase shift signal in the $\mathrm{Zr}-\mathrm{O}$ zones are very similar for each frequency. In the oxygen-enriched 
zirconium region, the concentration of the oxygen produces gradual modifications in the metal properties and implicitly in the SMM phase response. The size of the perturbed zone is about $18 \mu \mathrm{m}$ for all the depths investigated here. This value is close to the length of diffusion found on the EDS measurements considering that this last is apparently shortened by the surface pollution effect. At different depths under the scanned surface the spatial variation of the material properties is similar. The changes in the properties result only from the quantity of oxygen dissolved in the zirconium lattice.

One can also observe that the value of the phase shift increases with the frequency. The deeper one looks, the lower the phase shift.

Fig. 4e-f present the topography and the phase shift of the pure $\mathrm{Zr}$ zone. The phase shift signal shows no gradient, as expected. This observation confirms that the SMM technique is only influenced by the chemical composition of the sample.

Using several frequencies one can obtain information at various depths. To compare the information between the signals obtained at various frequencies, a procedure of normalization is applied. Scale and shift are chosen in order to obtain 0 value for the pure $\mathrm{Zr}$ and 1 for the $\mathrm{ZrO}_{2}$ zone. As remarked in Fig. 4 a, the topography is flat. The phase shift profile can only be the effect of changes in the material properties as seen in eqn (3) and is not influenced by the topography. The only parameter that changes along a line is the oxygen concentration. The local enrichment of the metal with oxygen is reflected in the phase shift measurements obtained by SMM. The location of the metal-oxide interface is given by the sudden change in the phase shift signal. The location given by SMM is very close to the location obtained on the topographic picture. This procedure can be used in cases where the location of the interface is not possible on the topographic image.

In the case of zirconium we know that at the oxide-metal interface the oxygen concentration should reach the solubility value, i.e. 29 at.\%. The oxygen concentration decreases progressively from $29 \%$ at the interface to 0 at. $\%$ in the pure metal zone.

According to eqn (1) the microwave signal penetration depends on the frequency used for the investigation: the lower the frequency, the deeper the penetration. Fig. 5 presents the results obtained at three different depths: $6.8 \mu \mathrm{m}, 3.9 \mu \mathrm{m}$ and $3 \mu \mathrm{m}$, i.e. respectively $2.21 \mathrm{GHz}, 6.87 \mathrm{GHz}$ and $11.83 \mathrm{GHz}$ along a straight line that contains $\mathrm{ZrO}_{2}$, the oxide-metal interface and $\mathrm{Zr}-\mathrm{O}$ zones.

The phase shift profiles obtained for three different frequencies, i.e. at three different depths, are almost superposed, as can be seen in Fig. 5. This shows that the phase curves are only influenced by the chemical composition of the solid solution. We recall here that the normalization is performed using only information on the pure $\mathrm{Zr}$ (which is set to zero) and $\mathrm{ZrO}_{2}$ (which is set to 1) and does not use the $\mathrm{Zr}-\mathrm{O}$ zone. The phase shift profile can be used to reveal the presence of oxygen and to measure its diffusion length.

Fig. 5 shows also the superposition of the SMM curves with the NRA measurements of the oxygen concentration in the $\mathrm{Zr}$.

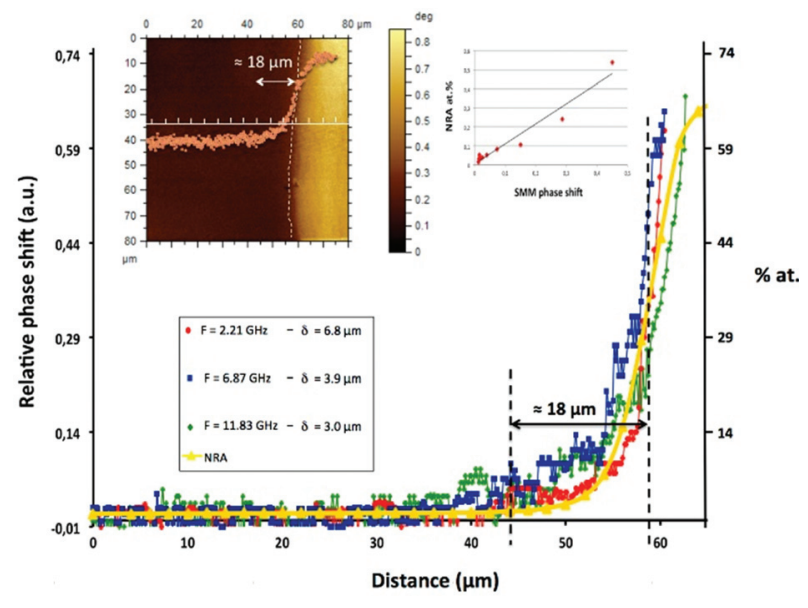

Fig. 5 Superimposed phase shift profiles for several depths with the location of the interface and compared with NRA results for the oxygen concentration.

One can remark that the SMM response shows that the size of the zone where the material has different properties compared to the original metal is sensibly the same as the oxygenenriched zone revealed by NRA.

The NRA results are obtained with a lateral resolution imposed by the accelerator beam size (about $3 \times 3 \mu \mathrm{m}^{2}$ and a depth of $3 \mu \mathrm{m}$ ) for a step scan size of $2 \mu \mathrm{m}$ between each measure point. Also this measurement represents an average value. By SMM, the lateral resolution is superior, since between two measure points the step scan size is $100 \mathrm{~nm}$ for $20 \mathrm{~nm}$ contact radius. This difference can explain the variations between the SMM curves and the NRA curve.

Moreover, on the SMM curves, we observe small change in the interface location for different investigation depths, as previously remarked in Fig. 4.

In addition, the phase shift $\varphi$ in SMM seems to be proportional to the oxygen concentration $c$ as follows:

$$
\varphi=K(c) c
$$

with $K(c)$ the proportionality factor which can be a function of the concentration. One can also propose the inverse form of the previous relation:

$$
c=F(\varphi) \varphi
$$

with $F(\varphi)$ the proportionality factor to be found by calibration. After calibration, eqn (9) can allow the measure of the oxygen concentration by SMM. This assumption of a proportionality relationship between the shift phase of SMM and the concentration of oxygen is confirmed when comparing the NRA measure with SMM measures. This rapid calibration, with the present measurements, shows a linear trend (Fig. 5, top right side vignette).

Consequently, under the $\mathrm{ZrO}_{2}$ layer, the SMM technique reveals a gradient of the metal properties. This gradient is related to the quantity of oxygen dissolved in the metal lattice. This method shows very encouraging results for the chemical 
characterization of oxygen solid solutions by permitting the visualization of the oxide-metal interface and allowing the measure of the diffusion length of the oxygen in the zirconium lattice with a spatial resolution of about $150 \mathrm{~nm}$.

\section{Conclusion}

In conclusion, the present work shows that the SMM technique not only allows the detection of sub-surface defects but also permits the detection of a gradient of properties produced by the presence of dissolved oxygen in the material. The possibility to characterize the in-depth distribution of the light chemical elements like oxygen dissolved in the metal represents the most important feature of the SMM technique presented here.

The great advantage of this technique compared to those currently used is that measurements can be realized under the contaminated surface without a specific sample preparation. The oxygen-enriched zone can be clearly identified.

The SMM phase shift profile can also show the location of the oxide-metal interface. The use of this technique as a tool for in-depth interface location is thus possible. One can also notice that the characterization can be made using different frequencies and realizing the cartography of the oxygen dissolved in the metal lattice at different depths under the scanned surface.

Thus, the SMM technique is a good candidate for in-depth measurements of local chemical enrichment. Moreover, we noticed that the phase shift response seems to be proportional to the oxygen concentration measured by NRA. The calibration of the SMM phase signal with respect to the concentration of the chemical element dissolved in the metal lattice could lead to a quantitative technique with sub-micronic spatial resolution. The authors are presently working on the calibration of the SMM method by comparing several oxygen concentration profiles measured by NRA with the corresponding SMM phase shift response.

\section{Acknowledgements}

The authors thank the financial support of the Conseil Regional de Bourgogne, Institute Carnot ARTS, LabeX Action "Integrated Smart System", CNRS and European Regional Development Fund Feder.

The authors thank Dr Luc Lavisse (ICB UMR 6303 - Dijon FRANCE) for his help in accessing the NRA technique.

\section{Notes and references}

1 J. J. Leani, H. J. Sanchez, R. D. Perez and C. Pérez, Anal. Chem., 2013, 85, 7069-7075.
2 H. D. Fiedler, E. E. Drinkel, B. Orzechovicz, E. C. Leopoldino, F. D. Souza, G. I. Almerindo, C. Perdona and F. Nome, Anal. Chem., 2013, 85, 10142-10148.

3 A. De Giacomo, R. Gaudiuso, C. Koral, M. DellAglio and O. De Pascale, Anal. Chem., 2013, 85, 10180-10187.

4 L. Luhl, I. Mantouvalou, I. Schaumann, C. Vogt and B. Kanngießer, Anal. Chem., 2013, 85, 3682-3689.

5 G. Binning, H. Rohrer, C. Gerber and E. Weibel, Scanning Tunneling Microscopy, Springer, 1993, pp. 31-35.

6 G. Binnig, C. F. Quate and C. Gerber, Phys. Rev. Lett., 1986, $56,930$.

7 J. C. Weeber, E. Bourillot, A. Dereux, J. P. Goudonnet, Y. Chen and C. Girard, Phys. Rev. Lett., 1996, 77, 53325335.

8 J. Krenn, A. Dereux, J. Weeber, E. Bourillot, Y. Lacroute, J. P. Goudonnet, G. Schider, W. Gotschy, A. Leitner and F. Aussenegg, Phys. Rev. Lett., 1999, 82, 2590-2593.

9 Y. Martin and H. K. Wickramasinghe, Appl. Phys. Lett., 1987, 50, 1455-1457.

10 D. Rugar, H. Mamin, P. Guethner, S. Lambert, J. Stern, I. McFadyen and T. Yogi, J. Appl. Phys., 1990, 68, 11691183.

11 G. Meyer and N. M. Amer, Appl. Phys. Lett., 1990, 57, 20892091.

12 M. Nonnenmacher, M. oBoyle and H. Wickramasinghe, Appl. Phys. Lett., 1991, 58, 2921.

13 C. Williams, Annu. Rev. Mater. Sci., 1999, 29, 471-504.

14 N. C. Bruce, A. Garcia-Valenzuela and D. J. Kouznetsov, Phys. D: Appl. Phys., 2000, 33, 2890.

15 S. Xu and M. F. Arnsdorf, Proc. Natl. Acad. Sci. U. S. A., 1995, 92, 10384-10388.

16 A. J. Huber, D. Kazantsev, F. Keilmann, J. Wittborn and R. Hillenbrand, Adv. Mater., 2007, 19(17), 2209-2212.

17 L. Tetard, A. Passian and T. Thundat, Nat. Nanotechnol., 2009, 5, 105-109.

18 G. Mills, J. Weaver, G. Harris, W. Chen, J. Carrejo, L. Johnson and B. Rogers, Ultramicroscopy, 1999, 80, 7-11.

19 J. H. Lee and Y. Gianchandani, Rev. Sci. Instrum., 2004, 75, 1222-1227.

20 R. Garcia and E. T. Herruzo, Nat. Nanotechnol., 2012, 7(4), 217-226.

21 C. Plassard, E. Bourillot, J. Rossignol, Y. Lacroute, E. Lepleux, L. Pacheco and E. Lesniewska, Phys. Rev. B: Condens. Matter, 2011, 83, 121409.

22 T. Hayes, M. Kassner, D. Amick and R. Rosen, J. Nucl. Mater., 1997, 246, 60-69.

23 L. Raceanu, V. Optasanu, T. Montesin, G. Montay and M. François, Oxid. Met., 2013, 79, 135-145.

24 E. Betzig, A. Lewis, A. Harootunian, M. Isaacson and E. Kratschmer, Biophys. J., 1986, 49, 269.

25 E. A. Ash and G. Nicholls, Nature, 1972, 237(5357), 510. 